\title{
European Society for Evolutionary Biology 2nd Congress
}

\section{Roma, Italy - September 3-7, 1989}

Details of the Congress organization, e.g. Symposia, plenary lectures, etc. have not been fully established. Congress circulars will be addressed to all ESEB members as well as to other interested people.

Correspondence: Professor Valerio Sbordoni

Dipartimento di Biologia

II Università di Roma "Tor Vergata"

via Orazio Raimondo - La Romanina

I-00173 Roma, ITALY 